\title{
Prevalence and Risk Factors of Adverse Drug Reactions Associated Multidrug Resistant Tuberculosis Treatments in Selected Treatment Centers in Addis Ababa Ethiopia
}

\author{
Haregewoin Bezu1 ${ }^{*}$, Daniel Seifu1, Getnet Yimer², Tesfamariam Mebrhatu ${ }^{3}$ \\ ${ }^{1}$ Department of Medical Biochemistry, College of Health Sciences, Addis Ababa University, Addis Ababa, Ethiopia \\ ${ }^{2}$ Research and Technology Transfer, College of Health Sciences, Addis Ababa University, Addis Ababa, Ethiopia \\ ${ }^{3}$ All Africa Leprosy, TB Rehabilitation and Training Center, Armauer Hansen Research Institute (AHRI/ALERT), \\ Addis Ababa, Ethiopia \\ Email: " haregewoin.bezu@gmail.com, daniel.seifu@aau.edu.et, getnetyimer@yahoo.com, \\ tesfameb@yahoo.com
}

Received 18 June 2014; revised 24 July 2014; accepted 6 August 2014

Copyright (C) 2014 by authors and Scientific Research Publishing Inc.

This work is licensed under the Creative Commons Attribution International License (CC BY). http://creativecommons.org/licenses/by/4.0/

c) (i) Open Access

\section{Abstract}

Introduction: The key to successful elimination of tuberculosis (TB) is treatment of cases with optimum chemotherapy. Irrational anti-TB drug use over time has led to drug-resistant TB. The treatment of MDR-TB with second line drugs is long, complex and costly, and has a considerable rate of adverse effects. The level of ADR reporting is low in Ethiopia due to different factors. This Study conducted in a selected treated area in Addis Ababa, Ethiopia and helped the health care centers to understand the prevalence of ADR related MDR-TB and be aware of those adverse effects in order to detect them early and be prepared to take proper steps when they occur. Aim of the Study: To determine the prevalence and risk factor of adverse drug reactions associated treatments of Multidrug Resistant tuberculosis. Method: This was a cross sectional study, which was conducted between March 2012 and February 2013 at St. Peter TB specialized hospital and AHRI/ALERT. 73 MDR TB patients, who were on MDR TB treatments, enrolled to the study. Adverse Drug Reactions associated MDR TB treatments were assessed by patient history review and questionnaire. Chemistry laboratory was used to test renal function, thyroid function, liver enzyme and potassium level. Result: In 72 patients, at least two ADRs were found. The mean age of the study population (Mean \pm SD) was $28 \pm 8.8$. In this study the most commonly found adverse

${ }^{*}$ Corresponding author.

How to cite this paper: Bezu, H., Seifu, D., Yimer, G. and Mebrhatu, T. (2014) Prevalence and Risk Factors of Adverse Drug Reactions Associated Multidrug Resistant Tuberculosis Treatments in Selected Treatment Centers in Addis Ababa Ethiopia. Journal of Tuberculosis Research, 2, 144-154. http://dx.doi.org/10.4236/jtr.2014.23018 
drug reactions (ADRs) were: Anorexia 83.3\%, Nausea and vomiting 82\%, Gastritis $64 \%$, Arteralgia $47 \%$, Skin rash and itching $45 \%$, Headache $29.2 \%$, Depression $22.2 \%$ and Blurred vision $19.4 \%$. Using binary logistic regression model older age (COR 8.71, 95\% [CI] 1.06 - 71.9), alcoholism (COR 4.05, 95\% [CI] 1.05 - 15.6), smoking (COR 0.24, 95\% [CI] 0.06 - 0.87) and concomitant drug intake (COR 0.14, 95\% [CI] 0.03 - 0.76) were independent predictors for ADRs. Conclusion: The prevalence of ADRs related MDR TB treatments is high. To minimize ADR occurrence, ADR predictors should be integrated into the clinical pathway. Monitoring of liver function, renal function, TSH and level of potassium during MDR TB treatment, helps to avoid complication caused by therapy and increase the adherence to the treatment.

\section{Keywords}

MDR TB, ADR, Second Line Anti-TB Treatment

\section{Introduction}

Tuberculosis (TB) is considered as a "global emergency" by the World Health Organization (WHO). According to the Ministry of Health Hospital statistics data, tuberculosis is one of the leading causes of morbidity, the fourth cause of hospital admission, and the second cause of hospital death in Ethiopia [1].

Anti-TB therapy includes a long-time, wide spectrum of drugs depending on the characteristics of the TB infection; new cases, re-infection, relapses, failures, Multi-Drug Resistant TB (MDR-TB) or Extensively DrugResistant TB (XDR-TB). Overall, this situation can predispose patients to develop adverse drug reactions due to the long-time exposition to the anti-TB drugs [2] [3].

Ethiopia, which ranks seventh on WHO's list of 22 high burden TB countries $\left(\mathrm{HBC}_{\mathrm{S}}\right)$ globally, is one of three countries in Africa with more than 5000 estimated new MDR-TB infections annually. Multidrug-resistance TB defined as resistance to at least isoniazid and rifampicin was also reported in about $1.2 \%$ of new cases and $12 \%$ of re-treatment cases. The Ethiopian government has identified MDR-TB as one of priority public health problems and it is committed to initiating comprehensive treatment for MDR-TB cases in the country [1] [4].

\subsection{Management and Treatment of Patients with Multi-Drug Resistant Tuberculosis in Ethiopia}

Multi-drug Resistant TB is simply TB disease that cannot be cured with the most effective drugs to date, i.e. isoniazid and rifampicin. Diagnosis cannot be confirmed with sputum, clinical picture or chest X-ray (CXR) but only from drug susceptibility testing (DST) [5].

According to FMOH 2009, in Ethiopia, during the initial phase, management of MDR-TB cases was coordinated by St. Peter TB Specialized Hospital in the city of Addis Ababa. It enabled St. Peter TB Specialized Hospital to function as a national referral centre in case of severe side effects and as a centre of excellence and training during the scaling up to the regions. Now days there are also other treatment centers emerged for initial phase of treatment.

Adverse drug reactions are a major cause of hospital admissions, but recent data on ADRs that develop following hospital treatment is lacking. Its development is a major public health problem contributing for a sizable percentage of admission, mortality, morbidity and increased health care costs. Lack of a system for monitoring drug safety is a major problem contributing to poor health in Ethiopia. Monitoring the safety of drugs contributes to building evidence on the safety of medicines pertinent to the Ethiopian population [1] [6]-[8].

The level of ADR reporting is low in Ethiopia due to different factors such as poor access to medical information, lack of enough awareness and training programs. Nationwide representative data on prevalence of MDR TB are lacking. This study conducted in a selected treated area in Addis Ababa, Ethiopia and helped the health care centers to understand the prevalence of ADR related MDR TB and be aware of those adverse effects in order to detect them early and be prepared to take proper steps when they occur and also helps policy decision makers [6] [7] [9] [10]. 


\subsection{Management of Adverse Drug Reactions Related to MDR TB Treatments}

Close monitoring of patients is necessary to ensure that the adverse effects of second-line drugs are recognized quickly by health-care personnel. The ability to monitor and diagnosis of patients for adverse effects is one of the major advantages of DOT over self-administration of DR-TB treatment based on Table 1 [1] [13].

Most ADRs can be managed with over-the-counter and common prescription drugs. If they are mild, continuing the treatment regimen, with the help of ancillary drugs where necessary is the best option. Many ADRs disappear or diminish with time and patients should be encouraged to tolerate the effects until they subside. Psychosocial support is an important component of management of ADRs. The most common ADR to second line anti TB drugs are Gastrointestinal Symptoms (nausea, vomiting, diarrhoea), skin reaction, ototoxicity, peripheral neuropathy, electrolyte wasting, psychiatric symptoms, nephrotoxicity, impaired vision and hypothyroidism [5] [11] [13].

\subsection{Risk Factors for Adverse Dug Reaction}

Many factors can increase the likelihood of an adverse drug reaction. This includes the simultaneous use of several drugs, very young or old age, pregnancy, and breastfeeding. Hereditary factors also make some people more susceptible to the toxic effects of certain drugs. Certain diseases can alter drug absorption, metabolism, and elimination and the body's response to drugs.

Taking several drugs, whether prescription or over-the-counter, contributes to the risk of having an adverse drug reaction. The number and severity of adverse drug reactions increase disproportionately as the number of drugs taken increases. The use of alcohol, which is technically a drug, also increases the risk [14].

Infants and very young children are at high risk of adverse drug reactions because their capacity to metabolize drugs is not fully developed. For example, newborns cannot metabolize and eliminate some antibiotic.

On the other hand older people are at high risk of having an adverse drug reaction for several reasons. They are likely to have many health problems and thus to be taking several prescription and over-the-counter drugs. Also, as people age, the liver is less able to metabolize many drugs, and the kidneys are less able to eliminate drugs from the body, increasing the risk of kidney damage by a drug and other adverse drug reactions [14] [15].

Sex is also an important determinant of drug use and drug response. Women tend to have a higher risk of adverse drug reactions with a 1.5 to 1.7 fold greater risk than men. Older age and female gender are significantly associated with ADR related hospital admission [16] [17].

\section{Materials and Methods}

Study area: This study was conducted from April 2012-March 2013 at St. Peter TB specialized hospital, high in the mountain of Entoto, North of the Ethiopia capital, Addis Ababa and at All Africa Leprosy, TB rehabilitation and training center/Armaur Hansen Research Institute (ALERT/AHRI) which is located in Kolfe Keranio Kifle Ketema, one of the research center in Addis Ababa.

Study population: The study population included MDR TB patients who were admitted and treated with second line anti TB treatments at St. Peter TB specialized hospital and (AHRI/ALERT).

Inclusion criteria: Age between 18 - 65 years, patients with Drug Sensitivity Testing (DST) confirmed MDR TB, and patients who signed written informed consent, were included.

Exclusion criteria: Critically sick patients (defined as those patients who needs management in an intensive care unit (ICU), patients who were not initiated (not started) anti MDR tuberculosis drugs, Patients receiving

Table 1. Laboratory monitoring of adverse drug effects of MDR TB treatment [11] [12].

\begin{tabular}{ll}
\hline \multicolumn{1}{c}{ ADR } & \multicolumn{1}{c}{ Frequency } \\
\hline Serum creatinine & Baseline and at least monthly while receiving an injectable drug. \\
Serum potassium & $\begin{array}{l}\text { At least monthly while receiving an injectable agent particularly those receiving capreomycin. } \\
\text { Thyroid stimulating hormone (TSH) }\end{array}$ \\
$\begin{array}{l}\text { Baseline. Ideally, once between months } 6 \text { and } 9 \text { of treatment, if receiving ethionamide and/or } \\
\text { PAS. Monitor for signs/symptoms of hypothyroidism. }\end{array}$ \\
$\begin{array}{l}\text { Consider periodic monitoring in patients receiving pyrazinamide for extended periods of time or } \\
\text { for patients at risk of hepatitis. }\end{array}$ \\
\hline
\end{tabular}


additional drugs known to cause ADR by forming drug-drug interaction, e.g. antiretroviral drugs and pregnant woman, were excluded from the study.

Statistical Analysis: SPSS version 20.0 was used for analysis of data. Average age was analyzed with mean \pm SE. Bivariate and multivariate analyses were used to test level of significance. OR and p-values were used to find the significant risk factors. Variables with $\mathrm{p}<0.05$ were considered as potential predictors of ADR.

Methods of data collection and analysis.

A total of 73 patients were included to the study. All patients were on MDR TB treatments. A structured questionnaire was used to facilitate the diagnosis of ADR, collection of data related to ADRS and risk factors such as age, sex, smoking, alcoholism and concomitant drugs. Retrospectively data also collected (reviewed) from patient's recorded chart in the study area. The dose, previous administration and use of any concomitant drug including over the counter medication or prescribed medication and herbs (including khat and shisha) were also assessed and reviewed. Headache, GI complicate, arthralegia, seizure etc were included in the questionnaires and the questionnaires filled by study subjects. Also possible adverse events were evaluated by symptom review.

SPSS version 20.0 was used for analyzed the data. Average age was analyzed with mean \pm SE. Bivariate and multivariate analyses were used to test level of significance. In this case ADR was the dependant variables whereas age, sex, concomitant drug intake, alcoholism and smoking were independent variables. OR and p-values were used to find the significant risk factors. Variables with $\mathrm{p}<0.05$ were considered as potential predictors of ADR.

Laboratory testing method

Sample collection

For chemistry laboratory investigation $4-5 \mathrm{ml}$ of venous blood was collected from the study subjects by trained persons. The sample was collected aseptically using regular or serum-separator vacationers and separated from the cell by standard laboratory techniques within $2 \mathrm{hr}$ of collection and freezed at $-20^{\circ} \mathrm{C}$ colder for longer storage. Tested enzymes and hormone include Alanine Aminotransferase (ALT) for liver function test, Creatinine Testing for renal impairment, Potassium level testing for hypokalemia and Thyroid Stimulate Hormone Testing for hypothyroidism.

\section{Results}

\subsection{Demography and Clinical Characteristics of the Study Population}

A total of 73 DST confirmed MDR TB patients were involved in the study. Age ranges from 18 - 65 with mean age (Mean \pm SD) of $28 \pm 8.8$. Among these, $25 \%$ were admitted to the inpatient and $75 \%$ were treated as outpatients. Majority of the patients $60 \%$ (44) were male and the remaining $40 \%$ (29) were female. Most of the patients (87.7\%) had a prior history of TB treatment (failure, default and relapse cases) and the remaining (12.3\%) had no prior history of TB treatment (Table 2). Among 73, two patients had extra-pulmonary tuberculosis (EPTB) and the remaining had Pulmonary TB (PTB). One patient who had EPTB was excluded from the study due to MDR TB drug allergy and this patient discontinues one or more therapy. None of the subjects were died due to ADR.

Second line drugs taken by the study subjects were capreomycin $(\mathrm{Cm})$, levofloxacin (Lfx), pyrazinamide (Z), ethionamide (Eto)/Prothionamide (Pto), ethambutol (E) and cycloserin (Cs). As shown Table 3, the drug is given based on the weight per $\mathrm{Kg}$ of the patients. Each drug cause more than two adverse drug reaction. After six months of the treatment the injectable (Cm) was stopped.

\subsection{Prevalence of Adverse Drug Reactions}

The total numbers of ADRs, found in this study, were 20. Gastrointestinal (GI) related adverse events were the most common ADR. Among the 72 patients, Anorexia was present in 83.3\% (60), Nausea and vomiting in 82\% (59), gastritis in 64\% (46), diarrhea in 9.7\% (7) and abdominal pain (cramp) in 6.9\% (5). Most patients developed GI disturbance early during treatment and almost all drugs cause GI symptom.

Other adverse drug reaction due to MDR TB drug commonly found in this study were decreasing hearing status present in 8.3\% (6), Arteralgia, in 46.6\% (34), Dermatological effect (Skin rash and itching), 37.5\% (27), Headache, 29.2\% (21), Blurry vision, 19.4\% (14), Dizziness, 13.9\% (10), Renal failure, 6.9\% (5), dehydration in 
Table 2. Demographic and clinical characteristics of the 73 patients treated with MDR-TB therapy.

\begin{tabular}{lc}
\hline \multicolumn{1}{c}{ Characteristics } & $\%(\mathbf{n})$ \\
\hline Gender & $60(44)$ \\
Male & $40(29)$ \\
Female & \\
Types of TB & $25(18)$ \\
$\quad$ Initiative phase (smear negative) & $75(55)$ \\
$\quad$ Continuous phase (smear positive) & \\
Diagnostic category of MDR TB & $87.7(64)$ \\
$\quad$ Previous treated with $1^{\text {st }}$ line medicine & - \\
Previous treated with $2^{\text {nd }}$ line medicine & $12.3(9)$ \\
Previously never treated for TB medication & \\
Characteristic of TB & $97.3(71)$ \\
PTB & $2.7(2)$ \\
EPTB &
\end{tabular}

Table 3. Summery of MDR TB medication received in individualized regimen.

\begin{tabular}{cc}
\hline Name of Drugs & Dosage $/ \mathbf{k g}$ \\
\hline Capreomycin (Cm) & $15 \mathrm{mg} / \mathrm{kg}$ \\
Levofloxacin (Lfx) & $500 \mathrm{mg}$ \\
Pyrazinamide (Z) & $20-30 \mathrm{mg} / \mathrm{kg}$ \\
Ethionamide (Eto)/Prothionamide (Pto) & $500-100 \mathrm{mg}$ \\
Ethambutol (E) & $15-20 \mathrm{mg} / \mathrm{kg}$ \\
Cycloserin (Cs) & $500-1000 \mathrm{mg}$ \\
\hline
\end{tabular}

5.6\% (4), Sleeping disturbance, 12.5\% (9), Psychosis, 1.4\% (1), Depression, 22.2\% (16) and Hypokalemia, 31.9\% (23). These ADRs were occurred at different points of time during the treatment (Table 4).

Screening for hypothyroidism was done clinically every 3 months. Screening for Hypokalemia and Renal impairment were done every month during the intensive phase and every 3-month during the continuous phase. In this study among 72 subjects only one patient had serum TSH level more than $10 \mathrm{mlu} / \mathrm{l}$. Hypokalemia was observed in $32 \%$ of patients on whom serum potassium level less than $3.4 \mathrm{mmol} / \mathrm{l}$ as obtained from laboratory result. In the case of RFT creatinine test was done and $6.9 \%$ of the patients shown serum creatinine level of greater than $1.5 \mathrm{mg} / \mathrm{dl}$. None of the participant had drug induced liver injury (ALT level < 2x ULN) which might be due to the removal of isoniazid and rifampicin which are the first line anti-TB drug that causes liver injury most frequently.

In this study anorexia, nausea and vomiting, gastritis, arthralgia, dermatological effects (skin rash and itching), hypokalemia, headache and depression were the most common Adverse Drug Reactions associated MDR TB treatment. Blurred vision, dizziness, burning sense of extremities, sleeping disturbance and hearing loss were common ADRs. Diarrhea, dehydration, renal failure, hypothyroidism and psychosis were less common ADRs. Liver injury and seizure were not found in the study.

\subsection{Risk Factors of Adverse Drug Reaction}

Associations that were assessed for potential factor in this study were gender, age group, concomitant drug intake (traditional medicine and herbs, prescribed and over the counter medication and others), smoking and alcoholism.

As shown in Table 5 below using a bivariate analysis, factor significantly associated with the occurrence of dehydration were those patients who were in the age group of $40-65$, (p-value $<0.05$ ) and crude odd ratio 
Table 4. Summary of total prevalence of Adverse Drug Reactions caused by MDR TB treatments in male and female subjects.

\begin{tabular}{|c|c|c|c|}
\hline Adverse Drug Reactions & $\begin{array}{l}\text { Total Number \% (n) } \\
\quad n=72\end{array}$ & $\begin{array}{c}\text { Female \% (n) } \\
n=28\end{array}$ & $\begin{array}{c}\text { Male \% (n) } \\
n=\mathbf{4 4}\end{array}$ \\
\hline Aneroxia & $83.3(60)$ & $75(21)$ & $88.6(39)$ \\
\hline Nausea and vomiting & $82(59)$ & $89.3(25)$ & $77.3(34)$ \\
\hline Gastritis & $64(46)$ & $64.3(18)$ & $63.6(28)$ \\
\hline Arthralgia & $47(34)$ & $57.2(16)$ & $38.6(17)$ \\
\hline Dermatological effect (skin rash and itching) & $37.5(27)$ & $42.9(12)$ & $34.1(15)$ \\
\hline Hypokalemia & $32(23)$ & $42.9(12)$ & $24(11)$ \\
\hline Headache & $29.2(21)$ & $39.3(11)$ & $22.7(10)$ \\
\hline Depression & $22.2(16)$ & $14.3(4)$ & $27.3(12)$ \\
\hline Blurred vision & $19.4(14)$ & $28.6(8)$ & $13.6(6)$ \\
\hline Burning sense of extremities & $15.3(11)$ & $25(7)$ & $9.1(4)$ \\
\hline Dizziness & $13.9(10)$ & $10.7(3)$ & $15.9(7)$ \\
\hline Sleeping disturbance & $12.5(9)$ & $17.9(5)$ & $9.1(4)$ \\
\hline Diarrhea & $9.7(7)$ & $7.1(2)$ & $11.3(5)$ \\
\hline Hearing loss & $8.3(6)$ & $3.6(1)$ & $11.4(5)$ \\
\hline Abdominal pain & $6.9(5)$ & 00 & $11.3(5)$ \\
\hline Renal failure & $6.9(5)$ & $3.6(1)$ & $9.1(4)$ \\
\hline Vaginal itching & $6.9(5)$ & $17.9(5)$ & - \\
\hline Dehydration & $5.6(4)$ & $10.7(3)$ & $2.3(1)$ \\
\hline Hypothyroidism & $1.4(1)$ & - & $2.3(1)$ \\
\hline Psychosis & $1.4(1)$ & - & $2.3(1)$ \\
\hline Seizure & - & - & - \\
\hline Liver injury & - & - & - \\
\hline
\end{tabular}

Table 5. Summary of risk factors associated Adverse Drug Reaction.

\begin{tabular}{|c|c|c|c|c|c|c|}
\hline \multirow{2}{*}{ Factors } & & \multicolumn{2}{|c|}{ Variables } & \multirow{2}{*}{ p-Value } & \multirow{2}{*}{$\begin{array}{c}\text { COR } \\
(95 \% \mathrm{CI})\end{array}$} & \multirow{2}{*}{$\begin{array}{c}\text { AOR } \\
(95 \% \text { CI })\end{array}$} \\
\hline & & Yes & No & & & \\
\hline \multicolumn{7}{|c|}{ Dehydration } \\
\hline \multirow{2}{*}{ Age } & $18-39$ & 2 & 52 & & \multirow{2}{*}{$8.71(1.06$ - 71.9) } & \multirow{2}{*}{$22.1(1.56-321)$} \\
\hline & $40-65$ & 2 & 7 & 0.04 & & \\
\hline \multicolumn{7}{|c|}{ Depression } \\
\hline \multirow{5}{*}{ Smoking } & Smoker & 6 & 7 & \multirow{2}{*}{0.03} & \multirow{2}{*}{$0.24(0.66-0.87)$} & \multirow{2}{*}{$0.3(0.04-2.26)$} \\
\hline & Non smoker & 10 & 49 & & & \\
\hline & & \multicolumn{2}{|c|}{ Sleeping disturbance } & & & \\
\hline & Smoker & 4 & 9 & 0.04 & $0.21(0.05-0.93)$ & - \\
\hline & Non smoker & 5 & 54 & & & \\
\hline \multirow[t]{2}{*}{ CD } & CDI & 3 & 4 & 0.03 & $0.14(0.03-0.76)$ & $0.50(0.04-8.95)$ \\
\hline & NCDI & 6 & 59 & & & \\
\hline \multicolumn{7}{|c|}{ Anorexia } \\
\hline \multirow[t]{5}{*}{ Alcoholism } & Drunker & 10 & 4 & 0.042 & $4.05(1.05-15.6)$ & - \\
\hline & Non drunker & 50 & 8 & & & \\
\hline & & \multicolumn{2}{|c|}{ Depression } & & & \\
\hline & Drunker & 7 & 7 & 0.009 & $0.184(0.06-0.65)$ & $0.25(0.04-1.8)$ \\
\hline & Non drunker & 8 & 50 & & & \\
\hline
\end{tabular}


(COR) 8.71 (95\% Confidence Interval [CI] 1.06 to 71.9). This study also assessed smoking status with different ADRs. Smoking was significantly associated with depression and sleeping disturbance $(\mathrm{p}<0.05)$ with COR of 0.24 (95\% Confidence Interval [CI] 0.66 to 0.8 ) and CRO of 0.21 (95\% Confidence Interval [CI] 0.05 to 0.93 ) respectively. Concomitant drug intake was significantly associated with sleeping disturbance with p-value of 0.03 and COR 0.14 (95\% [CI] $0.03-0.76)$.

Alcoholism was another risk factor that had significant association with sleeping disturbance and depression ( $<<0.05$ ) with COR of 0.14 (95\% Confidence Interval [CI] 0.03 to 0.76) and COR of 0.18 (95\% Confidence Interval [CI] 0.06 to 0.65 ) respectively. In the study, there were six subjects who were considered as alcoholics due to consume more than 2 units of alcohol per day. The alcoholic beverages include Beer, Areke and Draft.

\section{Discussion}

The main findings of this study were that of the 72 MDR TB patients who were on treatment developed two or more adverse drug reactions. Twenty different kinds of ADR (Table 4) were found in this study. Thus a variety of ADRs were found among patients receiving second line anti-TB drugs due to less efficiency of the treatment, which is in accordance with studies conducted previously [5] [17] which was reported that second line anti-TB drugs are less efficient, has serious side effects and more expensive than first line anti-Tb drugs. Other study also described that, patients who received second-line anti-TB therapy for MDR-TB present with a variety of side effects [18].

The onset of symptom of the adverse drug reaction was from 7 days after treatment initiation, which is similar to what was noted before [19] which described that the onset of ADR related MDR TB treatment was from 7 days to 120 days.

\subsection{Prevalence of Adverse Drug Reactions}

Adverse events such as GI related, Arteralgia, and Dermatological effects (skin rash and itching) were experienced by most of the patients in this study. Nausea and vomiting was present in 82\%, Epigastric pain in 64\%, Arteralgia in $47 \%$ and dermatological effect (skin rash and itching) in 37.5\%. Results of this study is in accordance with cohort study reported [20], which was found out that, nausea and vomiting present in $75 \%$ and Arteralgia in $47.1 \%$, which are stated as most common adverse drug reactions. Common MDR TB drug side effects such as Gastro intestinal complaint present in $79.2 \%$ (loss appetite, nausea, vomiting, diarrhea) and arthralgia in $64.6 \%$ were reported by other studies elsewhere [21].

Furthermore this study is also similar with studies [18] [19] [22] [23] described that most frequently observed side effects with MDR TB treated patients are GI disturbance, dermatological effect and arthralgia. In this study hearing loss also reported by $8.3 \%$ of the patients. Similar study also done that $18.75 \%$ of the patient developed sensorineural hearing loss involving in higher frequency [24] and hearing loss 6.3\% [21].

Hypokalemia is an electrolyte disturbance, one of the most challenging adverse reactions, which was assessed by using serum potassium determination, and present in $32 \%$ of the patients, found in this study. A retrospective case series study [25], hypokalemia is important adverse reaction of MDR TB therapy in which $31.3 \%$ (among 125 patients) had hypokalemia. In other 33\% of the patients developed hypokalemia, this is similar to our study [20]. Depression was present in $22.2 \%$ of the patient, however lower prevalence of depression observed in similar studies [19] [20].

Other common ADRs found in this study were blurry vision present in 19.4\%, burning sense of extremities $15.3 \%$ and headache in $29.2 \%$. A Kenyan study reported that headache and optic neuritis are nervous system related adverse drug reaction of MDR TB drugs [26].

In this study renal failure present in $6.9 \%$, dizziness in $13.9 \%$, sleeping disturbance in $12.5 \%$, and psychosis in $1.4 \%$ were less common ADRs. Similar study reported that, MDR TB drug side effects include psychosis $2.1 \%$ and blurry vision $6.3 \%$ [21]. A cohort Lativa study, also finds out that, renal insufficiency present in $4 \%$ and abdominal pain in 24\%, also common ADR related MDR TB therapy [23].

The reasons for heterogeneity in the prevalence of adverse events across the various study is unclear, but it might be related to several possible factors such as definition of adverse events, study design, demography, variation in the use of specific anti-TB drugs, pharmacokinetic or nutritional status. For instance in Ethiopia the standard regimen is E, Z, Km (Am), Lfx and Cs for 6 months and E, Z, Lfx, Eto and Cs for 12 months, whereas other study reported that second line drugs include Am, Cs, Eto, Km, Ofx, PAS and PTH. Studies reported that 
drugs used for MDR TB patients are Am, Ofx, Eto, Cs and clarithromycin. The difference in the drug regimen between different areas (resource rich and poor area) may cause some difference in the prevalence of ADR [27] [28].

In this study, liver injury was not found, because first line anti-TB drugs such as Isoniazid and Rifampicin which are among the first line anti-TB drugs that commonly cause of liver injury are excluded. This finding is similar to studies reported previously, in which liver injury was not a common adverse event of MDR TB treatment among patients receiving MDR TB therapy [21] [29].

Vaginal itching was present in 6.9\% (5) of patients. Adverse effect of MDR TB drugs includes candidial vaginitis. Patients with vaginal candidiasis usually reported pruritus (itching) but symptom is not specific for vaginal candidiasis [26] [30]. Further studies are needed in Ethiopia to explain this finding.

Most likely drugs that cause gastrointestinal symptom such as Nausea and/or vomiting were Ethionamide, Pyrazinamide and Ethambutol. In this study patients took Eto, $\mathrm{Z}$ and $\mathrm{E}$ in the first 6 and next 12 months of treatment and they developed nausea and vomiting. A prospective Indian study [29], described that Eto and E were suspected drugs that cause most likely nausea and vomiting. Nausea associated with vomiting was ubiquitous during early weeks of therapy, while the majority of the patients were experience nausea and vomiting early in treatment. This symptom may prevent the delivery of adequate therapy and leads patients for further problem.

Kanamycin and Cm cause Hypokalemia, which is associated with persistent nausea and vomiting [30]. Diarrhea and vomiting caused by antituberculous agents can contribute to GI electrolyte loss [25]. Aminoglycosidases cause renal wasting of potassium and this cause electrolyte abnormality. Electrolyte disturbance particularly hypokalemia appears to occur more frequently with capreomycin. Indian study described that aminoglycosidase used in MDR TB patients also result in irreversible hearing loss and occurred at a median time of 16 weeks (6 23) [24] [29].

Psychiatric disturbance such as depression, anxiety and sleeping disturbance were most likely caused by Cs and Eto which occur early in treatment. FQ and Cs were most likely drugs that cause Psychiatric disturbance which occurred on the median of 8 weeks [29]. In this study patients took Cs on the first 6 months of treatment and most of the time they developed psychiatric disturbance until they stopped Cs.

Hypothyroidism has vague and non specific symptom and can easily be missed by clinician. It is caused by PAS and Eto [31]. In the study site PAS was a reserved drug for MDR TB treatment and patients mostly took Eto and only one patient developed hypothyroidism on whom the serum TSH level was $>10$ mlu/l. Hypothyroidism occurred at a median time of 16 weeks (12 - 29) [29].

Renal impairment and decreasing hearing status also caused by $\mathrm{K}$ and $\mathrm{Cm}$. $\mathrm{Cm}$ and $\mathrm{Z}$ were also likely drugs that cause Arteralgia, which is similar to related studies done elsewhere [29] and this study also shows that renal impairment occurred at median time of 16 weeks (8 - 24).

\subsection{Risk Factors of Adverse Drug Reaction}

In this cross sectional study older age group, smoking, alcoholism and concomitant drug intake were independently associated with adverse drug reactions of MDR TB treatments. Many factors can increase the likelihood of an adverse drug reaction which includes the simultaneous use of several drugs, very young or older age, pregnancy and breast feeding. Hereditary factor also make some people more susceptible to the toxic effect of certain drugs [14].

According to the result of this study, age group of 40 - 65 was a risk factor, significantly associated with dehydration $(\mathrm{p}<0.05)$. As people age, the liver is less able to metabolize many drugs and the kidneys are less able to eliminate drug from the body. These age-related problems are often made worse by malnourishment and dehydration, which tend to become more common as people age [14].

Alcoholism also a risk factor, that was associated with sleeping disturbance and anorexia (loss of appetite) (p value $<0.05$ ). Alcoholism is a risk factor of ADRs and cause hypersensitivity reaction. Patients who were consume more than two units of alcohol per day considered as alcoholics. One bottle of beer contains $4.5 \%$ of alcohol volume which is equal to 1.3 units. Consuming alcohol more than 2 units per day is considered as alcoholic [32] [33].

Depression and sleeping disturbance also significantly associated with smoking. Concomitant drug intake such as drugs including herbs, over the counter and prescribed drugs and also using khat, shisha, etc. that were taken by the patient previously or with MDR TB drugs, was significantly associated with sleeping disturbance. 
Simultaneous use of several drugs is a risk factor for several ADRs. On the other hand experimental and clinical studies have found that nicotine, an active ingredient of both shisha and cigarettes, not only triggers cardiovascular diseases, but also predisposes frequent users to various neuropsychiatric disorders [14] [34]. This study also showed that Female genders were higher in developing adverse drug reaction which is in accordance with studies conducted previously [16] [35] [36] described that female gender is considered to be more at risk of ADRs and have greater risk for developing adverse drug reaction.

Due to differences at pharmacokinetics \& pharmacodynamics level, women are at high risk for developing adverse drug reactions. In women, absorption, protein binding, volume of distribution, clearance and metabolism of drugs may differ due to hormonal influences on physiological functions. Since many women world-wide take oral contraceptives and drugs are likely influenced by estrogens and progestogens as they interact at various enzymes and receptors levels. This study showed that most ADRs were higher in female than male. However, the study failed to establish the association between gender and ADRs due to smaller sample size [16].

From the different life style, factors most reported ADRs were association with smoker, alcoholism and concomitant drug intake. In our study alcoholism, concomitant drug taker and smokers were very few and most ADRs could not establish significance difference.

\section{Conclusions}

Adverse drug reactions have implication not only for the patient, but also for the entire health care system. Patients who received second line anti-TB therapy for MDR-TB present with a variety of side effects. Side effects of MDR-TB treatment are very common and most of them can be successfully managed. If side effects are not treated, they promote mortality and morbidity. Almost all patients with MDR TB developed more than two ADR. This decreases patient's adherence to treatment.

The prevalence of ADRs related MDR TB treatments is too high. Gastrointestinal events are the most common adverse events and occurred early in treatment. This prevents proper delivery and use of the drug. Monitoring of liver function (liver enzyme test), renal function, TSH and level of potassium during MDR TB treatment, helps to avoid complication caused by therapy and increase the adherence to the treatment.

Older age, smoking, alcoholism and using concomitant drugs including over the counter or prescribed drugs are risk factors appearing to be more prone for ADRs. MDR-TB patients who are, older age, taking alcohol (alcoholism), concomitant drug taker and smoker should be closely monitored for treatment related adverse events.

\section{References}

[1] Federal Ministry of Health (2009) Guideline for Program and Clinical Management of Drug Resistant Tuberculosis.

[2] Kocfa, C.D., Alejandro, R.M., Sonia Guillen, B., Eduardo, V.S., Andrea, S.M., Alexandra, N.G., Wilmer, S.C. and Antonio, B.O. (2011) Factors Associated with Anti-Tuberculosis Medication Adverse Effects: A Case-Control Study in Lima, Peru. PLoS One, 6, e27610. http://dx.doi.org/10.1371/journal.pone.0027610

[3] DOTS-Plus Guidelines (2010) Revised National Tuberculosis Control Programme. Central TB Division, Directorate General of Health Services, Ministry of Health \& Family Welfare, Nirman Bhavan, New Delhi.

[4] Abate, G. (2002) Drug Resistant TB in Ethiopia: Problem Scenarios and Recommendation. Ethiopian Medical Journal, 40, 79-86.

[5] Ignacio, M. and Sandya, H. (2010) A Basis for the Clinical Management of Complicated MDR-TB Cases Summarise, Best Practice in the Often Lengthy and Complex Managemenet of Multidrug-Resistant Tuberculosis. Africa Health.

[6] Abiy, E., Getahun, G., Mengiste, M. and Assegid, M. (2011) Adverse Drug Reaction in Ethiopia, Analysis of Case Reports, 2002-2007. Ethiopian Journal of Health Development, 25, 168-173.

[7] Yimer, G., Aderaye, G., Amogne, W., Makonnen, E., Aklillu, E., Lindquist, L., et al. (2008) Anti-Tuberculosis Therapy Induced Hepatotoxicity among Ethiopian HIV Positive and Negative Patients. PLoS ONE, 3, e1809.

[8] PLoS ONE (2009) Study Reveals High Level of Adverse Drug Reactions in Hospitals. February 11, 2009.

[9] Saukkonen, J.J., Cohn, D.L., Jasmer, R.M., Schenker, S., Jereb, J.A., Nolan, C.M., Peloquin, C.A., Gordin, F.M., Nunes, D., Strader, D.B., Bernardo, J., Venkataramanan, R. and Sterling, T.R. (2006) Hepatotoxicity of Antituberculosis Therapy. American Journal of Respiratory and Critical Care Medicine, 174, 935-952. http://dx.doi.org/10.1164/rccm.200510-1666ST

[10] Tostmann, A., Boeree, M.J., Aarnoutse, R.E., de Lange, W.C., van der Ven, A.J. and Dekhuijzen, R. (2008) A Antituberculosis Drug-Induced Hepatotoxicity: Concise Up-to-Date Review. Journal of Gastroenterology and Hepatology, 23, 
192-202.

[11] TB Policy, AFA (2011) Aids for Africa Health Care Professional Newsletter, June 2011-Issue 32. National Department of Health Guideline on the Management of Drug Resistant TB.

[12] World Health Organization (2008) Guidelines for the Programmatic Management of Drug-Resistant Tuberculosis. An Emergency Update. WHO/HTM/TB/2008.402, Geneva.

[13] TB Policy Guidelines (2004) DOTS-Plus for Standardized Management of Multidrug-Resistant Tuberculosis in South Africa Policy Guidelines.

[14] Joan, B.T. (2007) Risk Factors for Adverse Drug Reactions: The Merck Manual Home Health Hand Book.

[15] Knotek, M. (2013) Drug Induced Kidney Injury. Electronic Journal of IFFC, 20.

[16] Mahajan, S., Tandon, V.R. and Kuma, S. (2012) Women and Risk for Developing ADR's. JK Science, 14, 1.

[17] Dominica, C., Cristian, D., Niculae, G., Radu, P. and Constantin, M. (2011) Individualized Treatment Regimens with Second Line Anti-TB Drugs (SLD) Compared to First Line Anti-TB Drug (FLD) Only in New Pulmonary TB Patients, Culture Confirmed with Full Sensibility at Drug Sensibility Test (DST). Thematic Poster Session, 4406.

[18] Serena, K. (2010) Management of Side Effects during MDR-TB Treatment Partners. In Health: Brigham and Women's Hospital USAID TB CARE II.

[19] Shahina, Q., Iftekhar, A., Saifullah, B. and Nadeem R. (2011) Adverse Events in the Treatment of Multi Drug Resistant TB. Thematic Poster Session, 4402.

[20] Shin, S.S., Pasechnikov, A.D., Gelmanova, I.Y., Peremitin, G.G., Strelis, A.K., Mishustin, S., Barnashov, A., Karpeichik, Y., Andreev, Y.G., Golvbchikova, V.T., Tonkel, T.P., Yanova, G.V., Yedilbayev, A., Rich, M.L., Mukherjee, J.S., Furin, J.J., Atwood, S., Farmer, P.E. and Keshavjee, S. (2007) Adverse Drug Reaction among Patients Being Treated for Multi Drug Resistant TB Cohort in Tomsk, Russia. The International Journal of Tuberculosis and Lung Disease, 11, 1314-1320.

[21] Sin, C.I. (2011) Multi-Drug Resistant TB Drug Side Effect Management.

[22] Torun, T., Güngör, G., Ozmen, I., Bölükbaşi, Y., Maden, E., Biçakçi, B., et al. (2005) Side Effects Associated with the Treatment of Multidrug-Resistant Tuberculosis. The International Journal of Tuberculosis and Lung Disease, 9, 13731377.

[23] Bloss, E., Kuksa, L., Holtz, T.H., Riekstina, V., Skripconoka, V., Kammerer, S. and Leimane, V. (2010) Side Effects in Multi Drug Resistant TB Cohort in Lativa. The International Journal of Tuberculosis and Lung Disease, 14, $275-281$.

[24] Duggal, P. and Sarkar, M. (2007) Audiologic Monitoring of Multi-Drug Resistant Tuberculosis Patients on Aminoglycoside Treatment with Long Term Follow-Up. BMC Ear, Nose and Throat Disorders, 7, 5.

[25] Sonya, S., Jennifer, F., Felix, A., Anne, H., Keith, J., Epifanio, S. and Michael, R. (2004) Hypokalemia among Patients Receiving Treatment for Multi Drug Resistant Tuberculosis. Chest, 125, 974-980.

[26] Evans, A. (2008) Multi-Drug Resistant Tuberculosis, a Challenge in the Management of Tuberculosis. African Journal of Health Sciences, 15, 6-13.

[27] Waseem, S., Arshad, N. and Jamal, A. (2009) Retrospective Audit of Patients Treated for MDR-TB in Retreatment Category. Journal of Ayub Medical College Abbottabad, 21, 94-98. http://www.ayubmed.edu.pk/JAMC/PAST/21-2/Waseem.pdf

[28] Nathanson, E., Gupta, R., Huamani, P., Leimane, V., Pasechnikov, A.D., Tupasi, T.E., Vink, K., Jaramillo, E. and Espinal, M.A. (2004) Adverse Events in the Treatment of Multidrug-Resistant Tuberculosis: Results from the DOTS-Plus Initiative. International Journal of Tuberculosis and Lung Disease, 8, 1382-1384.

[29] Petros, I., Bhanumati, V., Homa, M., Helen, S.C., Joanna, L., Peter, S., Esdras, S., Samsuddin, K., Roma, P., Zarir, U., Giovanni, B.M., Giovanni, S. and Tony, R. (2012) Adverse Events among HIV/MDR-TB Co-Infected Patients Receiving Antiretroviral and Second Line Anti-TB Treatment in Mumbai, India. PLos ONE, 7, e40781.

[30] Owen, M.K. and Clenney, T.L. (2004) Management of Vaginitis. American Family Physician, 70, 2125-2132.

[31] Satti, H., Mafukidze, A., Jooste, P.L., McLaughlin, M.M., Farmer, P.E. and Seung, K.J. (2012) High Rate of Hypothyroidism among Treated for MDR TB in Lesotho. International Journal of Tuberculosis and Lung Disease, 16, 468-472. http://dx.doi.org/10.5588/ijtld.11.0615

[32] Marc, A.R. and Casillas, A.M. (2003) Adverse Drug Reaction: Types and Treatment Options. American Family Physician, 68, 1781-1791.

[33] National Health Service (NHS) Choices (2013) Alcoholic Unit. http://www.nsh.UK/livewell/Alcohol

[34] Abdullah, A.H. and Samir, A.A. (2002) Enemy within the Silent Epidemic of Substance Dependency in GCC Country. Sqc Journal for Scientific Research Medical Science, 4, 1-7.

[35] Redemarker, M. (2001) Female Patients Have Greater Risk for Developing Adverse Drug Reaction. American Journal 
of Clinical Dermatology, 2, 349-351.

[36] Zopf, Y., Rabe, C., Neubert, A., Hahn, E.G. and Dormann, H. (2008) Risk Factors Associated with Adverse Drug Reactions Following Hospital Admission: A Prospective Analysis of 907 Patients in Two German University Hospitals. European Journal of Clinical Pharmacology, 31, 789-798. 
Scientific Research Publishing (SCIRP) is one of the largest Open Access journal publishers. It is currently publishing more than 200 open access, online, peer-reviewed journals covering a wide range of academic disciplines. SCIRP serves the worldwide academic communities and contributes to the progress and application of science with its publication.

Other selected journals from SCIRP are listed as below. Submit your manuscript to us via either submit@scirp.org or Online Submission Portal.
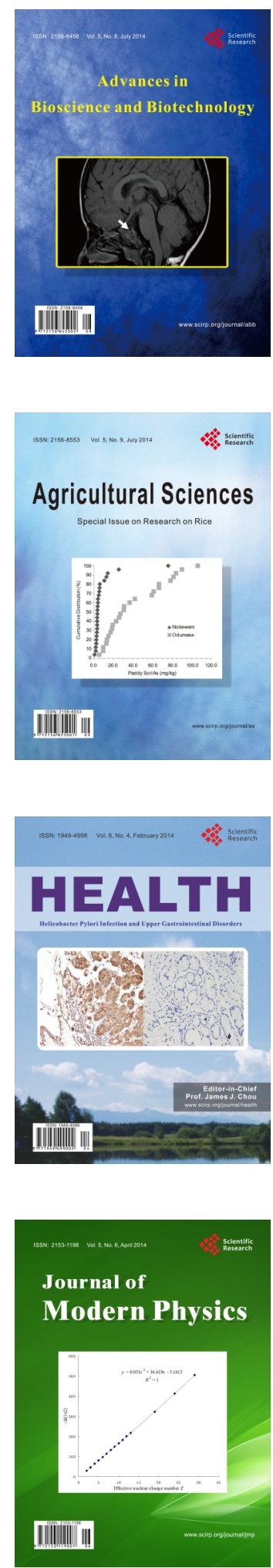
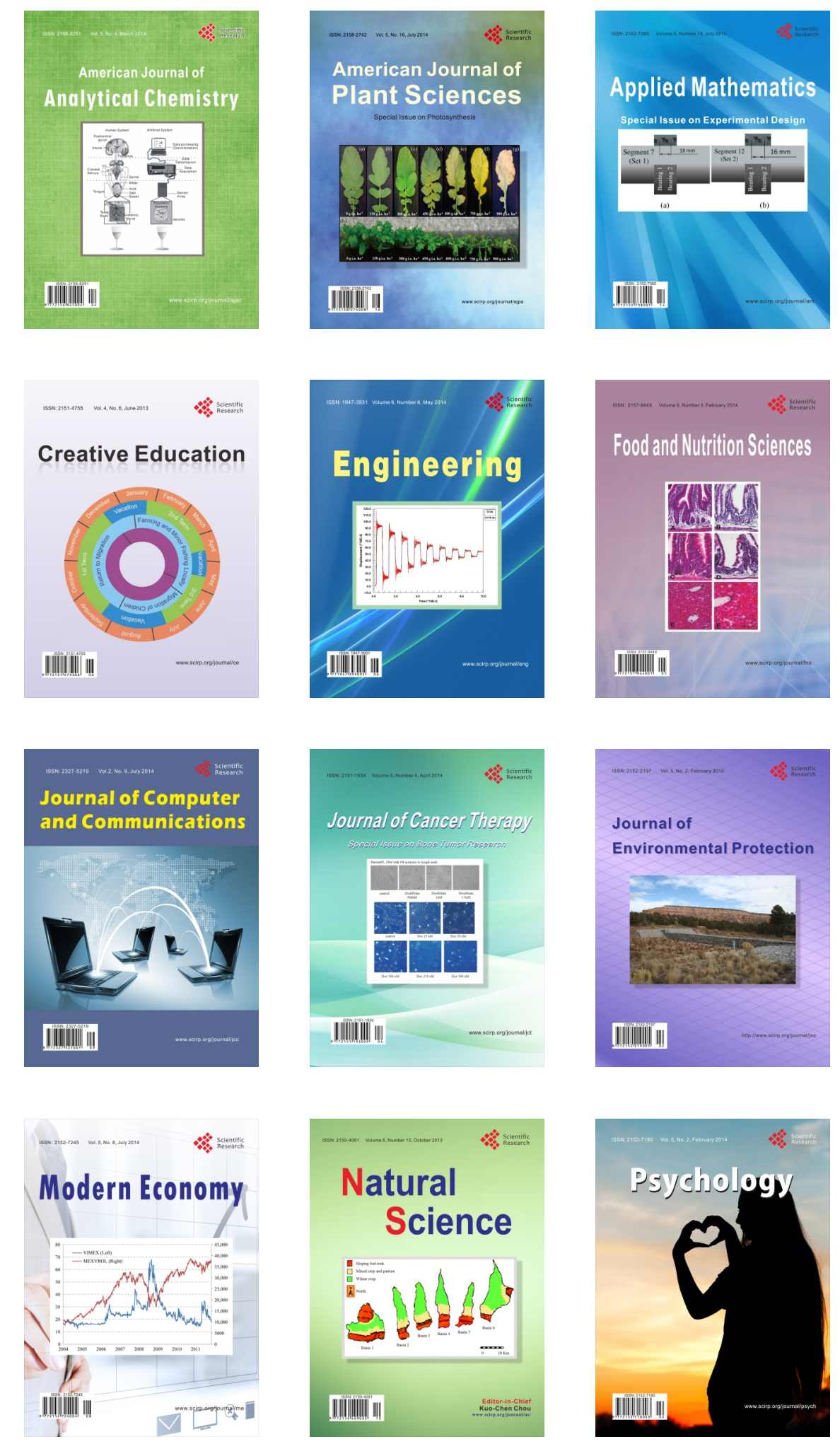